Meta

Journal des traducteurs

Translators' Journal

\title{
Entre la traduction interlinguale et intersémiotique. L'innovation dans les services de traduction vue par les responsables des entreprises de traduction françaises
}

\section{Anna Kuznik}

Volume 64, numéro 1, avril 2019

URI : https://id.erudit.org/iderudit/1065334ar

DOI : https://doi.org/10.7202/1065334ar

Aller au sommaire du numéro

Éditeur(s)

Les Presses de l’Université de Montréal

ISSN

0026-0452 (imprimé)

1492-1421 (numérique)

Découvrir la revue

Citer cet article

Kuznik, A. (2019). Entre la traduction interlinguale et intersémiotique. L'innovation dans les services de traduction vue par les responsables des entreprises de traduction françaises. Meta, 64(1), 194-214.

https://doi.org/10.7202/1065334ar
Résumé de l'article

L'innovation, comprise dans le sens large comme l'introduction de changements significatifs ayant pour but l'amélioration de la performance d'une organisation, a toujours été un défi méthodologique et social pour ceux qui voulaient la mesurer. Dans la discipline traductologique, un manque d'études panoramiques et exhaustives sur l'innovation dans les entreprises de traduction se laisse observer, malgré l'intérêt croissant des traductologues pour ces entités. Dans cet article, nous exposons les résultats d'une étude exploratoire (inductive, qualitative, descriptive) réalisée sur le territoire français en 2015. Dans notre approche, ce sont les personnes - les responsables des PME de traduction - qui constituent la source des données. Dans le cadre d'entretiens, elles ont partagé leurs opinions sur des éléments qui renforcent la possibilité d'émergence des innovations, sur les innovations proprement dites, et sur les formes créatives des services et de l'organisation du travail implantées dans leurs entreprises. Il ressort de ces milieux une conception traditionnelle de l'innovation, conçue presque uniquement comme un phénomène technologique, d'autres formes d'innovation n'étant pratiquement pas prises en compte. Le manque de formation initiale en entrepreneuriat, en gestion d'entreprise et en innovation est probablement l'une des causes. 


\title{
Entre la traduction interlinguale et intersémiotique. L'innovation dans les services de traduction vue par les responsables des entreprises de traduction françaises
}

\author{
ANNA KUZNIK \\ Uniwersytet Wrocławski, Wrocław, Pologne \\ anna.kuznik@uwr.edu.pl
}

\section{RÉSUMÉ}

L'innovation, comprise dans le sens large comme l'introduction de changements significatifs ayant pour but l'amélioration de la performance d'une organisation, a toujours été un défi méthodologique et social pour ceux qui voulaient la mesurer. Dans la discipline traductologique, un manque d'études panoramiques et exhaustives sur l'innovation dans les entreprises de traduction se laisse observer, malgré l'intérêt croissant des traductologues pour ces entités. Dans cet article, nous exposons les résultats d'une étude exploratoire (inductive, qualitative, descriptive) réalisée sur le territoire français en 2015. Dans notre approche, ce sont les personnes - les responsables des PME de traduction - qui constituent la source des données. Dans le cadre d'entretiens, elles ont partagé leurs opinions sur des éléments qui renforcent la possibilité d'émergence des innovations, sur les innovations proprement dites, et sur les formes créatives des services et de l'organisation du travail implantées dans leurs entreprises. Il ressort de ces milieux une conception traditionnelle de l'innovation, conçue presque uniquement comme un phénomène technologique, d'autres formes d'innovation n'étant pratiquement pas prises en compte. Le manque de formation initiale en entrepreneuriat, en gestion d'entreprise et en innovation est probablement l'une des causes.

\begin{abstract}
The measurement of innovation — viewed in a broad sense as an introduction of significant changes to improve company or societal performance-has always been a methodological challenge. Even if Translation Studies scholars pay ever more attention to translation enterprises we notice a lack of large-scale and exhaustive studies on innovation in these market-oriented entities. In the paper we outline the most relevant results obtained from an exploratory, qualitative, inductive study carried out in 2015 in France. By applying a subject-based approach to measure innovation issues and conducting interviews with heads of translation enterprises we gathered data on their opinions on factors which enhance the emergence of innovations, on innovation itself, and on their creative solutions implemented in new services and work organisation. It seems that in these translation SME the traditional conception of innovation, understood almost solely as technological improvement, remains dominant, and that all other forms of innovation offered by service activity are still underestimated. Arguably, translators' lack of initial training in entrepreneurship, management and innovation could be one of the main causes.
\end{abstract}

\section{RESUMEN}

La innovación, definida de manera amplia como introducción de cambios significativos para mejorar la actividad de una organización, ha sido siempre un gran desafío metodológico y social para los que intentaban medirla. En la traductología se observa una importante laguna de estudios en torno a la innovación desarrollada por las empresas 
de traducción, aunque los traductólogos cada vez más muestran su interés por estas entidades. En el artículo se exponen resultados obtenidos en un estudio exploratorio llevado a cabo en 2015 en Francia. La estrategia metodológica empleada fue inductiva, cualitativa, descriptiva. Las entrevistas realizadas durante una encuesta con las responsables de varias PME de traducción sirvieron como fuente de datos para medir los aspectos relacionados con la innovación. Las responsables expresaron su opinión acerca de los factores que refuerzan el surgimiento de las innovaciones, acerca de las innovaciones propiamente dichas y de las distintas formas creativas que se habían implementado en los nuevos servicios y en la organización del trabajo de la empresa. Parece ser que en este sector de servicios lingüísticos predomina la concepción tradicional de ver la innovación casi exclusivamente como un fenómeno tecnológico, y que las demás formas de innovar no quedan suficientemente explotadas. Probablemente, la falta de formación inicial de los traductores en temas de emprendimiento, gestión empresarial e innovación sería una de las causas a tener en cuenta.

\section{MOTS CLÉS/KEYWORDS}

innovation, créativité, entreprise de traduction, service de traduction, enquête innovation, creativity, translation enterprise, translation service, survey innovación, creatividad, empresa de traducción, servicio de traducción, encuesta

\section{Introduction: questions de recherche}

Dans cet article, nous nous proposons de présenter les résultats partiels d'une étude exploratoire effectuée en 2015, qui précède notre étude principale envisagée dans les années 2018-2019. Les résultats partiels exposés dans cet article correspondent à l'une des trois dimensions de l'objet d'étude, c'est-à-dire à la problématique des innovations dans le secteur des entreprises de traduction, les deux autres dimensions étant la conceptualisation de l'activité de traduction et la structure des services de traduction ${ }^{1}$. Dans cette partie de l'étude exploratoire, nous cherchons à savoir si le développement innovant des entreprises de traduction va dans le sens d'un ajout à leur offre de traductions interlinguales traditionnelles, la traduction intralinguale et intersémiotique (Sütiste et Torop 2007; Kuznik 2018). Si c'est effectivement le cas, dans quelle mesure cet élargissement de l'offre tend-il à se produire? Quels sont les autres moyens d'innover et les stratégies de développement projetés par les responsables des entreprises de traduction, à part l'élargissement de l'offre des services sur les terrains de la traduction intralinguale et intersémiotique? Quelles sont leurs réactions par rapport au terme innovation, les associations d'idées que ce terme leur évoque? Les services basés sur des traductions intralinguales et intersémiotiques constituent-ils une solide promesse d'innovation pour l'avenir dans l'esprit des experts chargés de profiler le développement des entreprises de traduction? Dans les sections qui suivent, nous allons présenter les idées et opinions de huit représentantes d'entreprises de traduction français. Leurs avis croisés révèlent une image intéressante de cette problématique.

\section{Cadre théorique}

Notre intérêt pour l'innovation dans les services de traduction a pris quelques années avant de se cristalliser sous la forme d'une étude exploratoire concrète (Kuznik 2014, $\left.2015 \mathrm{a}^{2}, 2015 \mathrm{~b}\right)$. C'est un sujet qui, évidemment, s'écarte des centres d'intérêt habituels 
de la traductologie, car il relève plutôt de la gestion d'entreprise et des disciplines économiques et organisationnelles.

\subsection{Objectifs, définitions, types et mesures de l'innovation}

Dans cette section, nous commençons par une présentation générale de quelques classifications des innovations, avec leurs objectifs et définitions, à savoir la proposition de l'Organisation de coopération et de développement économiques (OCDE/ Eurostat 2005) 3 , puis le modèle dit des «4P» de Bessant et Tidd (2007). Vers la fin de cette section, nous abordons la problématique de la méthodologie à appliquer pour mesurer les innovations.

\subsubsection{Classification de l'OCDE}

Pourquoi les sociétés cherchent-elles à innover? Dans une perspective de marché, la réponse que l'OCDE donne à cette question est empruntée à Tirole (1995): «[...] les entreprises innovent pour conserver leur compétitivité existante et pour trouver de nouveaux avantages en termes de compétitivité» (cité dans OCDE/Eurostat 2005: 30). Suivant cette idée, l'objectif de l'innovation dans le monde des affaires est d'amé-

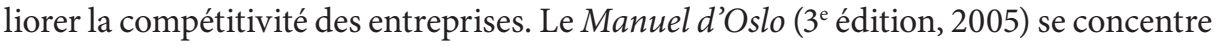
sur l'innovation dans les entreprises à vocation commerciale, c'est-à-dire les secteurs (primaire, secondaire et tertiaire) ${ }^{4}$ orientés vers le marché, mais reconnaît qu'elle peut aussi se manifester dans le secteur public, à vocation non commerciale, tels les services publics, la santé et l'enseignement. Au sens large, les innovations sont des changements planifiés et significatifs en vue d'améliorer la performance commerciale ou sociétale d'une entité. Plus spécifiquement, dans les secteurs commerciaux:

[...] une innovation est la mise en œuvre d'un produit (bien ou service) ou d'un procédé nouveau ou sensiblement amélioré, d'une nouvelle méthode de commercialisation ou d'une nouvelle méthode organisationnelle dans les pratiques de l'entreprise, l'organisation du lieu de travail ou les relations extérieures. (OCDE/Eurostat 1992/2005: 46)

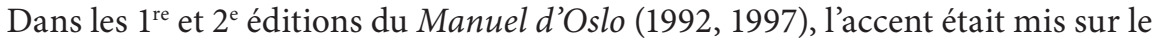
développement technologique de nouveaux produits et techniques de production par les entreprises, et l'innovation n'était donc définie que du point de vue technologique, ne portant que sur les produits et les procédés. La $3^{\text {e }}$ édition (2005) reconnaît deux nouveaux types d'innovations non technologiques, qui portent sur l'organisation et la commercialisation. Elle revoit aussi les deux premiers types (produits et procédés), puisqu'elle considère maintenant qu'une innovation peut se manifester dans l'amélioration significative non seulement d'un bien, mais aussi d'un service. La $3^{\mathrm{e}}$ édition reconnaît donc «l'importance de l'innovation dans des domaines où la $R \& D$ est moins intensive, tels les services et la production industrielle de faible technologie» (OCDE/Eurostat 1992/2005: 11).

Les auteurs sont très conscients de la spécificité des services en tant qu'activité économique et signalent que dans les secteurs orientés vers les services, l'innovation peut différer considérablement de celle des secteurs orientés vers la production industrielle, car les premiers ont «souvent une organisation moins formelle, de nature plus progressive (non radicale) et moins technologique» (OCDE 1992/2005: 11). Les services ont plutôt tendance à être un processus continu, et dès lors, l'identification d'une innovation sous forme d'événement ponctuel y est très difficile (OCDE 1992/2005: 38). 


\subsubsection{Modèle des 4P de Bessant et Tidd}

Le modèle des 4P de Bessant et Tidd (2007), qui classe les innovations suivant quatre types (produit, processus, position, paradigme), offre un puissant outil d'analyse des secteurs d'activité sociale. Partant de l'hypothèse que l'innovation réussie vise essentiellement la réalisation d'un changement positif, il met en avant quatre grandes catégories dans lesquelles peut s'inscrire ce changement:

- Innovation liée aux produits: changements au niveau des objets (produits ou services) proposés par une organisation. La forme d'innovation la plus courante est celle qui met au point ou améliore un produit ou service: un changement dans ce qui est proposé aux utilisateurs finaux.

- Innovation liée aux processus: changements dans les modes de création des produits et de prestation des services.

- Innovation sur le plan de la position: modifications du contexte dans lequel les produits ou services sont formulés et présentés.

- Innovation liée au paradigme: changements au niveau des modèles mentaux sousjacents qui influencent ce que fait l'organisation.

Kuznik (2014: 7) cite des exemples issus des actions humanitaires pour chaque type d'innovation dans le modèle des 4P de Bessant et Tidd (2007).

\subsubsection{Mesure de l'innovation: approche objet et approche sujet}

Mesurer l'innovation dans les services représente un défi. Dans ce domaine, en effet, les innovations sont souvent de nature tacite et immatérielle, associées à des connaissances individuelles (plutôt qu'à des connaissances générales inhérentes aux équipements et systèmes). Elles sont donc forcément désincarnées et non technologiques. Les formes traditionnelles de mesure de l'innovation (les départements R\&D des entreprises, les brevets), qui correspondent à ce que l'on appelle l'approche objet (interroger des spécialistes ou consulter des revues spécialisées pour identifier des annonces de produit) sont remplacées par de nouvelles formes correspondant à l'approche sujet (interroger les entreprises, et plus précisément, le personnel de ces entreprises).

$\mathrm{Vu}$ que nous voulions connaître l'avis des représentants des services de traduction à travers un contact direct avec eux, nous avons suivi les indications concernant l'approche sujet. Or, dans l'enquête (Bessant et Davies 2007) ${ }^{5}$ sur les pratiques innovantes des entreprises menée par le Department of Trade and Industry (DTI) britannique, les nouvelles méthodes de mesure suivantes ont été appliquées. Nous évoquons ci-dessous deux stratégies.

Premièrement, la langue et la formulation de l'enquête sont restées simples, et le mot innovation a été soigneusement évité dans la première partie du questionnaire: les personnes ont été interrogées plutôt sur les changements intervenus au cours des trois dernières années dans les différents aspects de leur activité. Le terme innovation a été évité parce qu'il est censé avoir acquis une charge sémantique trop forte et aurait donc demandé une définition (et les problèmes que cela aurait créés auraient été aussi nombreux que les solutions obtenues), et parce que l'on a tendance à associer les innovations à des faits ponctuels, ce qui pourrait les rendre difficiles à identifier dans les services, où le changement est vraisemblablement plus continu ou plus vague (Bessant et Davies 2007: 43). 
Deuxièmement, l'enquête a cherché à étendre l'investigation au-delà des limites étroites des innovations en termes de produit, de procédé ou de prestation, en explorant les changements intervenus dans l'entreprise en termes de technologies, de compétences, d'organisation et de relations avec la clientèle et les autres entreprises. Les auteurs ont ajouté ces aspects afin de comprendre l'innovation (ou le changement) dans un contexte plus large, plutôt que de comptabiliser des innovations et de comparer des pourcentages d'innovation.

\subsection{Les entreprises de traduction sous l'examen traductologique}

Depuis les premiers textes académiques qui rendaient compte de la situation de la traduction au sein des entreprises de traduction (Gouadec 1976), l'attention des traductologues s'est de plus en plus concentrée sur ces milieux de production des traductions. Avec les années, les chercheurs ont acquis une expérience méthodologique pour s'approcher de ce monde assez clos, et ont mis en œuvre des méthodes de collecte de données. Les premiers entretiens (Hermans et Lambert 1998) et enquêtes (Golden, Hurtado Albir et al. 1992; ACT 2005'; Dam et Zethsen 2009; Christensen et Schjøldager 2016), se voient enrichis par l'auto-observation (Kuznik et Verd 2010), l'observation (Risku 2014), l'analyse des sites Web des entreprises de traduction (Kou 2017) et par des méthodes mixtes (dans lesquelles les approches qualitatives et quantitatives sont intégrées) qui combinent l'analyse documentaire, l'entretien et l'observation (Kuznik 2010, 2012), ou encore l'enquête et le groupe de discussion (Presas, Cid-Leal et al. 2016).

Évidemment, les sujets abordés dans des études menées au sein des entreprises de traduction sont extrêmement variés et leur énumération dépasse largement les objectifs du présent article. À titre d'exemples, nous pouvons citer les approches ergonomiques (Lavault-Olléon 2011; Ehrensberger-Dow et Massey 2014), le contenu des postes de travail des traducteurs internes (Kuznik 2010, 2012; Kuznik et Verd 2010), la cognition située (Risku 2014), la traduction automatique et la post-édition (Carl, Bangalore et al. 2016), la localisation (Alonso 2014), et un service émergent appelé transcréation (Pedersen 2017).

La position des entreprises de traduction, leur réception et leur image dans le monde académique sont assez ambiguës: certains chercheurs soulignent l'existence des conflits entre ces entreprises et les traducteurs indépendants (Abdallah 2012), d'autres mettent en avant leur rôle crucial sur le marché de la traduction et, par là même, dans la société, en relevant plutôt les côtés positifs de leur activité (Gouadec 2002, 2007; Kuznik 2010, 2012, 2014). Il ne faut pas oublier cependant que ce sont ces entités qui assurent le lien entre les traducteurs indépendants et les clients, et de ce fait, elles ont une vision de la situation équilibrée, partagée entre les deux points de vue. Les PME de traduction sont aussi les entités les plus actives dans la recherche et le partenariat avec les universités (ACT 2005; Kuznik 2010, 2012; Presas, Cid-Leal et al. 2016).

Cette brève revue des études traductologiques consacrées aux entreprises de traduction indique que le thème de l'innovation n'a pas été fréquemment abordé jusqu'à présent, et surtout, qu'il n'a fait l'objet d'aucune étude large et complète. 


\section{Collecte et analyse des données}

Les données de notre étude exploratoire ont été collectées durant un séjour de recherche d'un mois à l'Université Grenoble Alpes en 2015. Après avoir effectué un échantillonnage circonstancié 7 , nous avons interviewé au total huit responsables dans cinq PME de traduction du département de l'Isère (tableau 1). Les cinq entretiens ont été transcrits et analysés grâce au logiciel EdEt (Kuznik, Verd et al. 2016). Pendant le traitement des fichiers transcrits, nous avons gardé les marques de l'oralité des entretiens (hésitations, répétitions, structures incomplètes, etc.) pour préserver l'authenticité de ces énoncés et pour pouvoir en analyser les caractéristiques langagières (Kuznik soumis[b]). Pour garantir l'anonymat des participants, mais aussi pour agrémenter la lecture et l'appréhension des résultats, les entreprises apparaissent dans cet article sous des noms de planètes. À la fin de l'article, nous proposons plusieurs champs d'applications possibles des résultats obtenus.

TABLEAU 1

Détail du matériel collecté et analysé

\begin{tabular}{|c|c|c|c|c|c|}
\hline Entreprise $^{*}$ & Vénus & Mars & Saturne & Neptune & Pluton $^{8}$ \\
\hline Fondation & 2004 & 2000 & 2005 & 2007 & $\varnothing$ \\
\hline Salariés & 6 & 9 & 3 & 2 & 10 \\
\hline Forme juridique & $\varnothing$ & sarl & scop & scop & $\varnothing$ \\
\hline $\begin{array}{l}\text { Durée de } \\
\text { l'entretien (min) }\end{array}$ & 45 & 50 & 50 & 30 & 40 \\
\hline $\begin{array}{l}\text { Nombre de mots } \\
\text { dans le fichier } \\
\text { transcrit }\end{array}$ & $\begin{array}{c}4507 \\
(25782)^{\dagger}\end{array}$ & $\begin{array}{c}6544 \\
(36753)\end{array}$ & $\begin{array}{c}5014 \\
(29378)\end{array}$ & $\begin{array}{c}2034 \\
(11021)\end{array}$ & $\begin{array}{c}3835 \\
(22184)\end{array}$ \\
\hline $\begin{array}{l}\text { Lignes de } \\
\text { transcription } \\
(\text { logiciel } E d E t)\end{array}$ & $1-244$ & $248-472$ & $474-622$ & $624-784$ & $787-1007$ \\
\hline \multirow{2}{*}{$\begin{array}{l}\text { Personnes } \\
\text { interviewées } \\
\text { (identifiant) }\end{array}$} & PM_Venus & \multirow{2}{*}{ Dir_Mars } & \multirow{2}{*}{ Coop_Saturn } & Coop1_Neptune & Dir_Pluto \\
\hline & TC_Venus & & & Coop2_Neptune & CD_Pluto \\
\hline \multirow{2}{*}{$\begin{array}{l}\text { Personnes } \\
\text { interviewées } \\
\text { (fonction) }\end{array}$} & $\begin{array}{l}\text { Responsable } \\
\text { des projets }\end{array}$ & \multirow{2}{*}{ Gérante } & \multirow{2}{*}{$\begin{array}{l}\text { Gérante (une } \\
\text { associée) }\end{array}$} & \multirow{2}{*}{$\begin{array}{l}\text { Les deux } \\
\text { associées }\end{array}$} & Gérante \\
\hline & $\begin{array}{l}\text { Coordinatrice } \\
\text { technique }\end{array}$ & & & & $\begin{array}{c}\text { Directrice } \\
\text { commerciale }\end{array}$ \\
\hline
\end{tabular}

* Identifiant par ordre dans le système solaire

$\varnothing^{\varnothing}$ Non mentionné lors de l'entretien

${ }^{\dagger}$ Nombre de caractères (espaces comprises)

\section{Résultats de l'étude exploratoire: le potentiel d'innovation}

Cette section présente les résultats obtenus dans la phase exploratoire de notre étude correspondant au potentiel d'innovation des services de traduction. Tout d'abord, nous abordons des points généraux d'identification des entreprises, de leur image commerciale et de l'entourage économique, c'est-à-dire tous ces éléments qui renforcent la possibilité d'émergence des innovations: normes, certifications, brevets, organismes professionnels, entourage institutionnel (la participation des entreprises interviewées dans cette recherche) et la situation dans le secteur de la traduction. 
Après ces informations contextuelles, l'essence du sujet sera traitée, à savoir les réponses de nos interlocutrices aux questions sur les innovations dans leur entreprise, la créativité au travail et les projets de croissance de l'entreprise dans l'avenir.

\subsection{Normes, certifications, brevets}

Au moment des entretiens, aucune des entreprises interviewées n'avait de certifications ni de brevets propres déposés, propres aux fournisseurs de services de traduction.

Pour Neptune et Saturne, les deux sociétés coopératives et participatives (scop, voir tableau 1), l'activité de traduction est marquée par une forte présence de la traduction assermentée et de l'interprétation pour les tribunaux, ce que nous pouvons considérer comme un certain label régulateur. En outre, ces deux sociétés sont parfaitement conscientes que leur taille les rend extrêmement fragiles (surtout Saturne, Coop_Saturn : 568) ${ }^{9}$.

Les autres sociétés (Vénus, Mars, Pluton) sont plus grandes, mais se trouvent en général trop petites et trop limitées en budget et en ressources pour acquérir des certifications. Par exemple, les responsables de Vénus soulignent à plusieurs reprises leur petite taille: "[Vénus] qui est une petite société» (PM_Venus: 8) et "Nous [Vénus], on est si petit» (TC_Venus: 10), ce qui apparemment explique le manque de certifications de leurs structures. En outre, les personnes interrogées sont d'avis que «les certifications ne sont pas apporteurs de business» (TC_Venus: 22).

Une remarque intéressante à propos de l'effectif de l'entreprise: Mars veut rester petite. C'est une attitude délibérée de sa part. La gérante explique ainsi cette décision:

On a décidé qu'on resterait dans la catégorie de moins dix, avoir toujours une dizaine de salariés, un peu moins [...] on a essayé de se former un tout petit peu au management et aux ressources humaines mais honnêtement, ce n'était pas notre métier, la production nous occupait vraiment beaucoup, donc on n'a pas franchi le cap, on est resté dans une structure de moins dix. (Dir_Mars: 254-258)

La gérante de Mars mentionne dans son propos un autre problème très important en tant que traductrice, à savoir le manque de formation en management et en gestion des ressources humaines. Nous reviendrons dans la suite de l'article sur cette question très liée à la petite taille des entreprises de traduction. Bien que Mars possède une structure de "moins dix», ses clients, quand ils visitent la société, sont contents de voir que les employés sont si nombreux (Dir_Mars: 270). La même société remarque que ses clients ne lui ont jamais demandé de présenter des certificats ou de suivre des normes particulières, et comme ces procédures sont coûteuses, l'entreprise a abandonné tout projet de se faire certifier par un organisme externe puisque ce n'était pas nécessaire (Dir_Mars: 262). Il est possible de respecter des procédures sans avoir de certifications, ce que fait Mars.

Pluton aussi est contente de sa «bonne réputation sur le marché» (Dir_Pluto: 867) et de remplir les conditions de base de la norme européenne ${ }^{10}$ et de la certification ISO ${ }^{11}$ Traduction, édition, relecture, mais suivant la gérante: «Ça ne suffit pas, si on ne met pas les bonnes personnes au bon endroit, on peut faire traduction, édition, relecture avec un mauvais résultat» (Dir_Pluto: 867).

Pluton se montre très critique envers les normes européennes ou ISO présentées comme des garanties de qualité. La directrice commerciale utilise une expression, 
« un flicage un peu ISO»(CD_Pluto: 861), pour signifier jusqu'à quel point le contrôle exagéré établi par la norme ISO repose, selon elle, sur un faux principe de garantie de qualité qui débouche en fait sur une situation absurde:

Dans ce contrôle, on assiste aussi de plus en plus à un flicage un peu ISO [...] qu'on doit donner systématiquement, de plus en plus les CV des traducteurs, ce qui ne veut rien dire, on fournit des tonnes de $\mathrm{CV}$ anonymes qui sont une absurdité [...] certains demandent le premier fichier de traduction, le deuxième fichier d'édition, ils demandent à voir les trois fichiers à chaque livraison, donc une espèce d'hyper contrôle qui ne garantit finalement rien, puisque c'est pas parce que sur un CV, le CV est bon que le traducteur est bon [...] il faut remplir des obligations, il faut dire: "regardez on a contrôlé, regardez, on est ISO, on correspond à la norme européenne”, ce qui était censé être positif devient un peu absurde. (CD_Pluto: 861)

La gérante de Pluton met ce contrôle excessif dans une perspective historique et l'explique aussi par le facteur nettement économique «des tensions sur les prix» (Dir_Pluto: 855-859). Quand nous lui avons demandé si l'entreprise possédait des brevets déposés, enregistrés, la gérante de Pluton nous a retourné la question: "Comment est-ce qu'on peut avoir des brevets enregistrés en traduction?» (Dir_Pluto: 885). Elle explique qu'actuellement, il n'est plus possible d'avoir de brevets dans ce secteur, car le marché est déjà trop mûr, saturé de nouveautés technologiques:

On arrive aujourd'hui à une certaine maturité technologique dans la traduction, c'est difficile de créer des produits innovants, aujourd'hui vous avez des outils d'aide à la traduction TAO, je dirais que tous les six mois on en a un nouveau. Et puis des aides gratuites. Il y en a plusieurs qui sont sortis au cours de la dernière année, qui sont gratuits. De même, le thème de la traduction automatique... [...] Donc il va y avoir sur le marché plein de choses [...] on était arrivé au bout de la phase de maturité et on s'approchait de la phase où on allait tomber parce que, oui, ça y est, on a un niveau d'automatisation, c'est quand même extrêmement important dans la traduction, le workflow, les outils de traduction, l'informatique [...] on est sur le plateau, il n'y a plus guère de nouvelles idées en ce moment. (Dir_Pluto: 889-909)

Mars et Pluton parlent toutes les deux à ce moment de nos entretiens d'une possibilité manquée d'avoir un brevet relatif à des logiciels de gestion de projets (évoqués ci-dessous dans une autre section). La gérante de Pluton avoue: "Alors je réponds "non" mais on aurait pu en avoir» (Dir_Pluto: 889). La directrice commerciale confirme qu'à l'heure actuelle, elle ne considère plus que ce soit possible, également pour des raisons de mondialisation et de concentration du capital. Même une société comme Pluton n'a pas de moyens pour développer les logiciels «jusqu'au bout» (CD_Pluto: 891) :

Je dirais qu'on ne peut plus [avoir des brevets] parce qu'il y a peut-être un changement d'échelle, ce que tu disais qu'il y a quinze ans le marché n'était pas mature donc en fait on aurait développé un logiciel de gestion, on pouvait le faire parce que ça n'avançait pas aussi vite, maintenant ça avance si vite qu'on n'a plus les moyens de concurrencer des sociétés qui ont cinquante développeurs en Hongrie, il nous reste très peu de moyens pour les développer jusqu'au bout. (CD_Pluto: 891) 


\subsection{Organismes professionnels mentionnés et projets de recherche en collaboration avec les universités}

Au cours de nos échanges, les responsables des entreprises de traduction françaises mentionnent très peu d'institutions ou d'organisations externes qui les accompagnent dans leur chemin vers l'innovation.

La gérante de Mars évoque une formation sur l'innovation mise en place par le Centre international de ressources et d'innovation pour le développement durable (CIRIDD) (Dir_Mars: 402). La coordinatrice technique de Vénus mentionne la Chambre nationale des entreprises de traduction (CNET), qui en principe devait l'aider à obtenir une certification (TC_Venus: 18). La même Chambre, avec la Société française des traducteurs (SFT), se voit critiquée par la gérante de Mars (Dir_Mars: 454).

Les universités sont également présentes dans l'environnement institutionnel de ces entreprises, ne serait-ce que par la réalisation des projets de recherche communs (recherche fondamentale ou appliquée) ${ }^{12}$. C'est pourquoi, durant les entretiens, nous avons voulu connaître les opinions de nos interlocutrices sur le type de recherche que nous étions en train de mener, sur les modalités de participation à adopter (confidentialité de l'information, anonymat, visibilité, etc.) et sur leur motivation. En général, l'accueil a été très positif et chaleureux. Il est vrai que notre échantillonnage s'est fait de manière circonstancielle, grâce aux contacts de la responsable de Master de traduction spécialisée de l'Université Grenoble Alpes, ce qui nous a garanti un bon accueil.

Dans ce contexte, la responsable de projets de Vénus affirme: «Moi personnellement, je trouverais ça plutôt bien, je trouve que c'est important de participer dans ce genre de choses" (PM_Venus: 154). La coordinatrice technique la suit dans cette conviction: "Je crois que c'est bien» (TC_Venus: 154). Elles se sont toutes deux visiblement préoccupées de la confidentialité de l'entretien, puisqu'elles se sont rassurées en constatant: «On n'a pas vraiment dit rien de confidentiel» (PM_Venus : 154), «On n'a pas parlé des choses strictement confidentielles» (TC_Venus: 162) et «C'est vrai on n'a pas trop parlé de nos clients» (PM_Venus: 160). Pour nous, en tant que chercheure, apprendre ce que ces entreprises considèrent comme confidentiel a aussi été un acquis très important. Pour la gérante de Pluton, sont considérés comme information confidentielle tous les services «autres que le transfert d'une langue à une autre» (Dir_Pluto: 935). La traduction audiovisuelle, le sous-titrage et les autres, «c'est confidentiel pour l'instant, on a des options pour en faire un peu plus mais ce n'est pas notre cœur de travail, de métier» (Dir_Pluto: 821), parce que «le marché est ouvert, c'est-à-dire sur les milliards qui sont brassés en traduction, $99 \%$ du transfert se fait d'une langue à une autre» (Dir_Pluto: 939).

La gérante de Mars nous a aussi expliqué pourquoi ses sentiments sont mitigés lorsqu'elle voit venir un chercheur: il a le pouvoir de valoriser ensuite (positivement ou négativement) l'information qu'il a recueillie:

Ça va dépendre de vos objectifs [...]. Tout dépend du contenu. Ce que je veux dire par là, c'est que c'est toujours intéressant de faire de la publicité, de se faire connaître, de développer son image, je ne vois pas de sociétés qui vont dire: "non, surtout ne parlez pas de moi" [...]. Mais après, oui, tout dépend de ce que vous dites dans l'étude: si dans votre étude vous dites: "surtout ne faites pas appel à cette société", forcément je n'ai pas envie que vous parliez de moi. (Dir_Mars: 406-414) 
Elle est cependant consciente que dans la recherche scientifique et académique, l'objectivité et l'impartialité sont de rigueur: "En même temps j'imagine très bien vos lecteurs qui disent: "Ah, mais de toute façon elle a été rachetée par une société, elle dit cela parce que ça intéresse la société." Quelle est alors la crédibilité de votre étude?»(Dir_Mars: 418).

À la question de savoir si nous pourrions citer sa société dans nos publications, la gérante de Saturne a répondu: "Je pense que c'est bien d'avoir les noms des participants» (Coop_Saturn: 580).

Chez Neptune, à notre question de savoir si elles ne voyaient pas d'inconvénient à être citées ou, au contraire, si elles préféraient rester anonymes, la première associée a dit: «Je pense qu'on peut être citées» (Coop1_Neptune: 742). La deuxième renchérit: «Oui, c'est pareil, je crois qu'il n'y a pas vraiment de mieux» (Coop2_Neptune: 744). Elle nous a signalé à ce moment que nous étions les premières chercheures à nous intéresser à Neptune et à leur demander des renseignements pour une recherche en cours (Coop2_Neptune: 738).

La directrice commerciale de Pluton a manifesté beaucoup d'intérêt pour l'objet de notre étude et son axe directeur (CD_Pluto: 981-985).

\subsection{Situation présente et future dans l'industrie de la traduction}

Saturne a une vision assez négative de la situation sur le marché de la traduction, marquée par la baisse des tarifs, la concentration du capital, les rachats de PME par de grands groupes, la mondialisation et les mémoires de traduction de mauvaise qualité: le récit entier de Saturne sur sa relation avec la revue de presse allemande en ligne, pour laquelle la société a travaillé de longues années, est un excellent exemple d'histoire commerciale marquée profondément par l'instabilité, les bouleversements, déplacements, restructurations, voyages, revalorisations des langues et des tarifs, remises en perspective globale des conditions de travail, etc. Le monde se trouve en constante transformation, semble vouloir nous dire la gérante de Saturne à chaque moment de l'entretien.

La gérante de Pluton partage avec nous ses observations sur la situation complexe, divergente, paradoxale du marché de traduction. Dans son expression, l'évolution de la réalité est toujours à double mouvement: les "d'un côté et de l'autre», «à la fois», "mais parallèlement» reviennent fréquemment dans les phrases où elle dévoile ses opinions sur la situation présente et future de l'industrie de traduction (voir aussi Kuznik, soumis[b]). Avec sa directrice commerciale, elle nous parle de la traduction automatique, de la consolidation du marché et de sa fragilisation (chacun peut s'autoproclamer traducteur), de la dévalorisation de la profession, et des tarifs sans cesse réduits (Dir_Pluto et CD_Pluto: 837-947).

Devant cette situation extrêmement complexe, la gérante de Mars déplore le manque d'esprit associatif, de communication, de partage (Dir_Mars: 372, 378). C'est pourquoi elle a choisi pendant notre entretien la planète Mars comme identifiant de son entreprise: elle veut batailler pour changer cette situation et mettre en valeur l'image du traducteur (Dir_Mars: 454). 


\subsection{Innovations}

\subsubsection{Réactions au sujet de l'innovation}

En général, nos interlocutrices ont été assez surprises que nous abordions avec elles le sujet des innovations. Nous nous sommes vite rendu compte qu'elles n'avaient pas l'habitude d'en parler.

La responsable de projets de Vénus nous a dit: "Je ne sais pas, c'est compliqué, c'est compliqué comme notion [...] c'est très peu concret; c'est très compliqué, oui» (PM_Venus: 122-150). Elle se montre réticente à parler de ce sujet, mais en même temps consciente de la complexité de cette notion, et met en relief dans son opinion le caractère évolutif des innovations dans le temps:

Comme maintenant en plus pour le moment on est en plein développement pour certaines activités, peut-être dans six mois on pourrait y voir plus clairement et parler des innovations. On aura peut-être mis en place certaines choses et là je pense qu'on n'a pas encore assez de recul, c'est pareil, il y a des choses qui fonctionnent bien actuellement, même si on a envie de mettre des choses en place, je ne sais pas si on pourrait parler d'innovation. (PM_Venus: 126)

Après nous avoir présenté ses récents projets technologiques, la gérante de Mars constate, comme pour clôturer ce sujet dans notre conversation: «Je ne sais pas si c'est vraiment très innovant, notre marque unique c'est vraiment la relation de proximité avec le client, une relation étroite qu'on crée avec nos clients, je ne crois pas que ce soit très innovant» (Dir_Mars: 394). La réaction de la représentante de Saturne va dans la même direction. Elle décrit ses projets de créativité textuelle, et ensuite, constate: "Je ne sais pas si toutes les entreprises le font» (Coop_Saturn: 572).

Les associées de Neptune suivent le sujet des innovations «très de loin» (Coop1_ Neptune: 718), car elles ont de bons clients institutionnels en Suisse et n'ont pas besoin d'innover.

Pour Pluton, le sujet des innovations est visiblement confidentiel. La responsable refuse d'en parler au cours d'un entretien de ce type:

Même si j'en [des innovations dans l'entreprise] avais, je ne vous le dirais pas [...] Ça paraît assez évident qu'on veut protéger ses innovations, oui on a des éléments, je pense, qu'on met en œuvre, que toutes les sociétés de traduction ne mettent pas en ouvre, mais si on les révèle... (Dir_Pluto, 895-899)

La gérante de Pluton veut protéger ses idées innovantes, mais aussi, elle est consciente du caractère évolutif des changements introduits sur le marché, comme dans le cas de la post-édition. Elle souligne le fait que les innovations émergent dans une chaîne, et les unes sont conséquences des autres:

En fait, dans la traduction, les idées se propagent vite et sont vite copiées. [...] Non, mais sur le marché il y a une immense innovation qui est la post-édition mais il faut un peu de temps pour que de grosses innovations provoquent de petits changements de pratiques par une onde de choc. (Dir_Pluto: 913-917)

\subsubsection{Exemples d'innovations}

Comme exemple de son innovation, Vénus cite une plateforme de gestion de projets de traduction installée sur le cloud (TC_Venus: 130-146). Pour cette société, à 
l'époque, cela a représenté un immense progrès car avant, elle se servait d'Excel pour sa gestion de projets.

La gérante de Mars décrit deux projets de type technologique, mais mentionne en même temps les obstacles qui ont freiné ces innovations, à savoir la taille et le budget: «[...] toujours avec nos petits moyens» (Dir_Mars: 374 ), «J'étais trop petite pour eux [développeurs d'un logiciel]» (Dir_Mars: 382) et «Oui mais c'est du budget, c'est des sous, c'est de l'argent» (Dir_Mars: 390). Son premier outil technologique a été un logiciel de gestion de projets (Dir_Mars: 370-386). Le deuxième outil technologique innovant mentionné par la gérante de Mars, c'était une plateforme pour référencer des traducteurs indépendants (Dir_Mars: 398).

Dans sa réponse sur les innovations, une des associées de Neptune fait une référence directe au Dragon et marque sa distanciation par rapport à la post-édition et à la transcréation (Coop2_Neptune: 724).

\subsubsection{Manque de formation initiale en gestion de l'entreprise et en innovations}

La gérante de Mars reconnaît qu'au début de son entreprise, en tant que traductrice, le manque de formation en gestion de l'entreprise et aux ressources humaines s'est fait ressentir (Dir_Mars: 254). Avec sa collègue, cofondatrice de l'entreprise, elles ont "essayé de se former un tout petit peu au management et aux ressources humaines mais honnêtement, ce n'était pas [leur] métier»(Dir_Mars: 258). Avec son esprit d'entreprise, elle s'intéresse cependant vivement aux formations (également absentes dans sa formation initiale de traductrice) dans le domaine de l'innovation:

Je viens de m'inscrire dans une formation mise en place par le CIRIDD, le Centre international de ressources et d'innovation pour le développement durable, et c'est une action collective pour l'économie de fonctionnalité, c'est-à-dire vraiment la transversalité, et je vais voir avec eux comment appliquer ça... ils font ça beaucoup dans l'industrie, mais comment appliquer ça aux services et comment j'espère pouvoir faire en sorte de proposer des choses innovantes dans ce domaine-là. (Dir_Mars: 402)

La gérante de Saturne, traductrice de formation, avoue qu'elle se pose des questions sur une possible formation pratique à l'interprétation, mais en plus, reconnaît le manque de formation en prospection:

C'est toujours la difficulté, on est tout le temps la tête dans le guidon, on répond toujours aux demandes, mais on n'a jamais vraiment d'opportunités pour la prospection, on n'a pas forcément de formation pour le faire, non plus. En 2016 ce sera l'objectif: élargir notre portefeuille. (Coop_Saturn: 504)

\subsection{Créativité au travail}

Les gérantes ne se sentent pas à l'aise lorsqu'elles parlent d'innovations. Elles préfèrent parler de créativité au travail. «Innovantes, pas forcément. [...] Je ne dirais pas innovantes, mais créatives en fait», avoue la représentante de Saturne (Coop_Saturn: 564-568). La directrice commerciale de Pluton précise: «On met en place toujours une création des nouveautés, d'approches peut-être pas révolutionnaires mais des nouveaux procès» (CD_Pluto: 901). La responsable de projets de Vénus souligne aussi le caractère évolutif des changements innovants dans la société (PM_Venus: 126; voir la section 4.4.1). 
Nous avons regroupé ci-après leurs interventions en deux blocs : la créativité mise à l'œuvre dans de nouveaux services, et la créativité présente dans de nouvelles formes d'organisation des services de traduction et de la vente.

\subsubsection{Créativité dans de nouveaux services}

Les deux associées de Saturne ont «développé le côté “créatif”"(Coop_Saturn: 568) grâce à des cursus qu'elles ont suivis et qui leur «ont permis de faire d'autres choses que de la traduction» (Coop_Saturn: 568). De plus, «cela joue dans [leur] style de rédaction ", trouve la gérante de Saturne (Coop_Saturn: 568).

À part cette créativité rédactionnelle chez Saturne, la gérante de Mars mentionne la créativité dans le contexte du service de transcréation, qu'elle appelle d'ailleurs la rédaction car elle trouve que la "[transcréation] c'est un néologisme qui n'est pas très heureux»(Dir_Mars: 290).

Elle souligne aussi que cette exigence de création marque la différence entre la rédaction et la traduction: "Quand on crée [dans le cas de la transcréation], on est beaucoup plus libre, et en même temps, même pour beaucoup de personnes, c'est difficile parce qu'elles ne se sentent pas capables de créer» (Dir_Mars: 298).

Les représentantes de Pluton ont mis de la créativité dans un de leurs nouveaux services appelé par la directrice commerciale «une sorte de coaching linguistique» (CD_Pluto: 829), qui se trouve entre la traduction et l'autre chose (CD_Pluto: 829-833).

\subsubsection{Créativité dans de nouvelles formes d'organisation et de vente}

Comme exemple d'application de la créativité dans l'organisation des services de traduction, la gérante de Saturne évoque leur organisation sous forme de correction mutuelle et de travail en binôme dans le cas des traductions vers la langue étrangère, la correction étant toujours assurée par une native (Coop_Saturn: 527).

En outre, la même gérante de Saturne voit plutôt leur innovation «dans le fonctionnement quotidien» (Coop_Saturn: 572), la scop étant organisée sur la base du télétravail (Coop_Saturn: 588). La gérante explique cette nécessité du télétravail par un facteur temporel, parce que la société a dû s'adapter à un rythme de travail très exigeant et restreint en termes de délais (572).

Comme mission de son entreprise de traduction, la gérante de Mars se propose d'être une linguiste d'affaires (Dir_Mars: 426) et de créer le service de traduction en amont du processus de ce service, en étroite collaboration avec le client. "Quand on me demande un service de traduction, c'est déjà trop tard», soupire-t-elle à un moment de notre entretien. Elle désire aussi optimiser la communication linguistique des sociétés qui l'entourent (Dir_Mars: 426). Dans la définition de la mission de l'entreprise, elle évoque une tendance à créer et à proposer des services personnalisés, suivant les besoins des clients (Dir_Mars: 310-314). Sa définition du traducteur dévoile également le défi de créer un service personnalisé: «Un traducteur, c'est quelqu'un qui comprend les besoins et qui fournit un service adapté; ça ne veut pas dire qu'il doit tout traduire, non, mais qu'il saura utiliser des outils» (Dir_Mars: 454).

La gérante de Saturne nous parle de son projet de regrouper des services autour du service de traduction, mais aussi d'inclure dans son offre tous les services annexes, nécessaires au client. Elle appelle cela une espèce d'offre globale (Coop_Saturn: 536) et de la communication d'entreprise (Coop_Saturn: 556). "Comme on travaille sur- 
tout avec des agences en Suisse [...] je pense donc qu'en 2016, on essaiera de développer plus les contacts sur ce marché en y proposant les services de ce type», constate la gérante (Coop_Saturn: 536).

\subsection{Projets d'évolution des entreprises de traduction interviewées}

Les réponses des responsables des entreprises de traduction interviewées au sujet de leurs projets de développement concrets pour l'avenir se mélangent à leurs opinions sur la possible évolution du marché des services de traduction en général. Cependant, nous avons pu relever certaines idées intéressantes, décrites ci-après.

\subsubsection{Développement technologique accru}

Le développement technologique se trouve au cœur des débats et des préoccupations dans l'esprit de chacune de nos interlocutrices. La croissance technologique de ces sociétés est fortement inscrite dans leur histoire, dès la naissance de ces structures. La responsable de projets de Vénus constate: "[Vénus] c'est une société qui a beaucoup évolué dans le sens qu'à la base [2004], on a commencé avec une commerciale et puis des chefs de projets et aujourd'hui [2015], on a aussi des personnes en technique» (PM_Venus: 8 ).

D'après la même responsable de projets, la tendance qui va grossir le marché de la traduction, et ce vers quoi évolue Vénus, ce sont les plateformes Internet sur lesquelles les traductions s'intègrent en ligne et pour lesquelles tous les intervenants du projet (le traducteur, le relecteur, et le relecteur en interne, le cas échéant) coopèrent (PM_Venus: 96).

Chez Saturne également (Coop_Saturn: 40), cette nouvelle voie de prospection sur des plateformes en ligne correspond parfaitement au souhait de celle-ci d'offrir des services regroupés, globaux, comme évoqué ci-dessus (voir section 4.6.2.).

Parmi les idées pour l'avenir, la coordinatrice technique de Vénus mentionne l'intégration obligatoire de la traduction automatique dans les processus de travail, surtout quand le client ne réclame pas la qualité de traduction la plus haute et que tendre vers celle-ci n'est donc pas nécessaire (TC_Venus: 102, 110).

En suivant l'évolution de la traduction automatique et de la post-édition, la gérante de Mars, comme celle de Saturne, n'est pas «très optimiste sur l'avenir des sociétés de traduction en général et des traducteurs au sens actuel du terme» (Dir_ Mars: 334). Pourtant, notre interlocutrice met en avant la question de l'existence d'un marché «à double vitesse» (Dir_Mars: 338), c'est-à-dire d'une situation dans laquelle il existe parallèlement des entreprises très avancées en termes d'outils technologiques et d'autres qui ne le sont pas du tout.

Sa vision de deux niveaux technologiques coexistants paraît être un écho de la vision d'un développement paradoxal, divergent, du marché de la traduction, évoquée plus haut par la gérante de Pluton. Heureusement, dans ce panorama extrêmement technologiste, il subsiste en même temps, pour la gérante de Mars (Dir_Mars: 338), " des domaines très rédactionnels, très spécifiques, où la création est importante, où est notre intérêt, sinon les machines vont prendre le dessus, pour moi en tout cas c'est certain». Parallèlement, la gérante de Pluton prophétise l'avènement prochain de «l'intelligence artificielle»: 
Mais où est-ce qu'on va aller [après la post-édition] ? Est-ce qu'on va aller plus loin? [...] La dernière génération de traduction automatique, c'est le réseau de neurones, l'intelligence artificielle, c'est en train de venir, c'est la nouvelle génération de traduction automatique. (Dir_Pluto: 919-923)

\subsubsection{Projets de prospection mitigés}

Après avoir entendu toutes ces opinions sur la situation générale du marché de la traduction, on peut avoir l'impression que le sort de ces PME interviewées est fatal, mais c'est loin d'être le cas. Les plus petites structures surtout (les deux scop) se déclarent contentes de leur situation. La gérante de Saturne est plutôt rassurante:

Nous sommes plutôt contentes [...]. Aujourd'hui ça fonctionne bien, on n'a pas de soucis de trésorerie, on n'a pas de mauvais payeurs, ce qui est la difficulté parfois, et notamment les tribunaux, mais cela semble s'arranger en termes de délai de paiement maintenant. (Coop_Saturn: 500-504)

Elle désire prochainement «élargir [son] portefeuille» (Coop_Saturn : 504) et proposer des services intégrés, globaux (voir plus haut, section 4.5.2).

Les deux associées de Neptune expriment une même satisfaction en parlant de leur travail (Coop1_Neptune: 718, 722; Coop2_Neptune: 646, 652, 704). Cependant, cette satisfaction actuelle doit être comprise dans le contexte d'une période difficile traversée précédemment (Coop1_Neptune: 690, 698; Coop2_Neptune: 696). Maintenant qu'elles ont redressé la barre, elles appliquent dans leur démarche une attitude ergonomique et cherchent le bien-être personnel: «Là on a enfin un équilibre, et maintenant notre projet était d'augmenter un peu nos salaires pour l'année prochaine» (Coop1_Neptune: 698); «l'idée c'est de gagner notre vie en de meilleures conditions de travail, d'être mieux nous»(Coop2_Neptune: 700), avouent-elles. Ainsi exprimé, l'avenir est donc plutôt incertain et complètement imprévisible:

D'après l'expérience qu'on a eue, on ne sait pas trop comment on va évoluer / En fait, j'ai du mal à dire ce qu'on fera dans l'avenir, les clients ne savent pas tellement les volumes qu'ils vont nous confier [...] on n'a pas vraiment fait un plan comme ça sur le futur, même de recruter quelqu'un d'autre, ça ne se fera pas tout de suite. (Coop2_ Neptune: 692 / Coop1_Neptune1:690)

Les associées de Neptune ont aussi eu une certaine réflexion sur leur développement à venir, mais ces idées ont été abandonnées ensuite, car finalement, elles n'y voyaient pas vraiment de sens (Coop1_Neptune: 702).

Par ailleurs, le volume considérable de traductions à réaliser au quotidien ne leur laisse pas de marge temporelle pour s'occuper de la prospection plus systématiquement, ce qui est souvent le sort des petites structures commerciales:

Puis il y a des nouveaux clients qui nous demandent de très gros volumes de travail / [...] effectivement, depuis beaucoup de temps on n'a pas eu une journée libre, c'est encourageant / [...] c'est vrai qu'on n'a pas trop de temps pour penser ce qu'on va faire, pour chercher des nouveaux clients, pour nous développer [...] pour l'instant oui, [ça tombe tout seul].(Coop2_Neptune: 704 / Coop1_Neptune: 708/Coop2_Neptune: 710) 


\section{Conclusions}

\subsection{Innovations}

Au cours de l'enquête, les responsables des entreprises de traduction françaises rencontrées ont décrit d'une manière détaillée les tendances et les tensions qui existent dans le secteur de l'industrie de la traduction. Leurs interventions ont touché la problématique des prix et des conditions de travail, des locaux et du télétravail, de la mobilité, du besoin d'optimiser la sous-traitance et le contact avec les clients, et évidemment, des technologies utilisées dans la production des services de traduction, des logiciels présents ou à venir, et de la relation générale entre l'homme et la machine (l'outil). Il faut interpréter ces témoignages comme propres à la situation des PME et des scop. Cette situation s'inscrit dans le contexte plus large de l'évolution divergente du marché, où il existe des mondes parallèles en ce qui a trait au développement technologique, et où les petites structures sont plutôt contentes, cherchent le bien-être personnel et construisent des projets de prospection modérés (tendance notée dans différents réseaux de traduction par Risku, Rogl et al. 2016: 18). Néanmoins, une certaine crainte relativement aux changements radicaux survenus dans le domaine de la traduction se manifeste dans les opinions des représentantes de ce secteur du marché.

Malgré la maturité technologique très avancée du secteur de la traduction et la taille plutôt petite des entreprises interviewées, nous avons pu remarquer la présence d'un esprit de créativité et d'une nette volonté de perfectionnement des services traditionnels de traduction. Cette créativité se voit engagée dans de nouveaux services: la créativité rédactionnelle, la transcréation, le coaching linguistique. Elle s'imprime aussi dans de nouvelles formes d'organisation et de vente: correction mutuelle et travail en binôme, télétravail, création du service avec le client en amont du processus, mission de conseil linguistique, individualisation des services, regroupement des services (reconnu comme une tendance innovante par Kwieciński, Młodzińska-Granek et al. 2015: 32-33), recherche de nouveaux marchés. Certes, la traduction audiovisuelle et la transcréation (alors des services qui rajouteraient à la traduction interlinguale des éléments intralinguaux et intersémiotiques) sont considérées comme des possibles voies de développement futur de l'entreprise, mais nos interlocutrices n'ont pas voulu nous donner de détails sur ces démarches pour des raisons de concurrence et de confidentialité.

Il nous paraît tout de même surprenant que nos interlocutrices méconnaissent la typologie classique des innovations et qu'elles se sentent si incertaines lorsqu'elles s'expriment à ce sujet. En revanche, elles se montrent beaucoup plus à l'aise en parlant de la créativité au travail. Leur manque de formation en gestion de l'entreprise est probablement à l'origine de cette incertitude. Cependant, il a été clair pour nous que, dans leurs opinions, la première façon de comprendre l'innovation, c'est celle qui met en relief les aspects technologiques (traduction automatique, logiciels, outils informatiques), ce qui est une façon traditionnelle de définir et de mesurer les démarches innovantes. Les services offrent un éventail plus large de solutions innovantes (qui ne se limitent pas aux innovations technologiques) et c'est bien dommage que ce champ ne soit pas suffisamment exploité dans les services de traduction. Malgré les efforts des organisations mondiales (par exemple de l'OCDE citée au début de cet article) de saisir l'essence des innovations «autres-que-technologiques », donc tacites, 
immatérielles, progressives et moins formelles, le secteur des PME de traduction ne perçoit que l'aspect technologique comme décisif et presque exclusif dans sa définition de l'innovation.

\subsection{Recherche engagée, pertinence sociale, compétitivité}

La méthodologie de collecte de données employée dans cette étude (entretiens semistructurés), typique de l'approche sujet de l'innovation, possède des traits communs avec la recherche engagée (RE, en anglais action research) par son caractère direct et collaboratif. Au cours des entretiens, nous avons soulevé des sujets jusqu'à un certain point nouveaux pour nos interlocutrices autour des innovations (les résultats présentés dans cet article) et de la classique division tripartite en traduction intralinguale, interlinguale et intersémiotique proposée par Jakobson en 1959 (Kuznik 2018, soumis[a]). Nos questions les ont invitées à une réflexion sur leurs activités et pratiques. Les interviewées et l'intervieweuse ont ainsi partagé et construit un savoir commun. Comme le remarquent Cravo et Neves (2007: 98-99), les praticiens sont souvent trop occupés par leurs défis professionnels au jour le jour et ne trouvent pas de mobilisation directe pour une réflexion.

Par là, dans cette étude, nous avons démontré une fois de plus que la séparation entre la théorie et la pratique est une fausse séparation (Gambier 1986, 2005; Cravo et Neves 2007: 97). Malgré le fait que notre étude ne correspond pas complètement à une RE (l'élément cyclique, répétitif, en spirale en étant la pièce manquante; Cravo et Neves $2007: 94$ ), elle peut être jugée socialement pertinente, et cela, sur les trois niveaux proposés par Gambier (2005: 5-6) : «a. Dans la définition des objectifs assignés à tel ou tel projet [...] b. Dans le programme même de la recherche et son organisation [...] c. Dans la dissémination, la diffusion des résultats.»

Cette collaboration participative reste toutefois subordonnée aux impératifs de compétitivité et de confidentialité caractéristiques de la réalité économique (Ehrensberger-Dow 2014; Kuznik 2016: 6-8). Les défis et les risques de conduire une RE en collaboration avec des entités commerciales privées sont plus élevés que dans le cas du secteur public (écoles, musées, etc.). L'accès aux entités privées reste tellement difficile que certains auteurs doutent qu'une recherche objective et rigoureuse avec ce type de partenaires soit possible, car ils ne sont pas disposés à s'ouvrir entièrement au chercheur (Presas, Cid-Leal et al. 2016: 135, 138). Partant de la perspective de la sociologie du travail, Durand (2004: 254-259) distingue trois niveaux d'entreprises: (1) «l'entreprise normative [qui] dit ce que doit être l'entreprise», (2) «l'entreprise normée, visible, celle que l'on visite et l'on étudie», et (3) «l'entreprise simulée, celle qui est invisible, celle qui se cache, celle qui ne veut pas être vue», le troisième niveau étant le plus intéressant pour le chercheur, mais jamais accessible.

\subsection{Champs d'applications}

La présente recherche peut s'appliquer aux champs suivants:

- en formation des entrepreneurs en traduction, concrètement en matière d'évaluation du risque, des formes de l'innovation, de la culture de l'innovation et de l'entrepreneuriat numérique; dans ce sens, les résultats de l'étude de Gieure et BuendíaMartínez (2016: 5381) indiquent que la formation en entrepreneuriat constitue l'un 
de deux facteurs cruciaux de la survie des entreprises de traduction sur le marché, la proximité des membres de famille entrepreneurs et des amis entrepreneurs dans l'entourage social des entrepreneurs en traduction (business background) en étant le deuxième facteur déterminant;

- en formation des traducteurs, comme source d'inspiration pour les activités de formation professionnalisée en traduction, comme c'était le cas par exemple dans le projet didactique de Galán-Mañas (2013);

- en transfert des connaissances, pour renforcer les liens entre l'académie et le marché, pour que les enseignants et les chercheurs en traductologie ne perdent pas le contact avec la réalité du secteur des services de traduction (Kwieciński, Młodzińska-Granek et al. 2015, dans leurs exemplifications des projets de transfert des connaissances relevant des sciences humaines et sociales en Allemagne et en Pologne ne citent aucune entreprise centrée sur les services de traduction);

- en marketing des services de traduction, car les opinions exprimées par les responsables des entreprises de traduction sont formulées dans un certain langage spécialisé qui est commun à ce secteur et qui peut aider à mieux organiser la vente et la distribution de ces services (Kuznik, soumis[b]);

- en gestion des entreprises de traduction, parce que cette étude recueille des témoignages d'expertes en la matière, en montrant les côtés forts et les côtés faibles, difficiles de cette activité.

\section{REMERCIEMENTS}

Nous remercions l'ambassade de France en Pologne et Sébastien Reymond, attaché de coopération scientifique et universitaire, ainsi que son équipe, pour nous avoir attribué une bourse du gouvernement français (BGF) pour un séjour de recherche d'un mois en novembre et décembre 2015 à Grenoble; Élisabeth Lavault-Olléon et le Groupe de recherche multilingue en traduction spécialisée (ILCEA4/ GREMUTS) pour nous avoir accueillie à l'Université Grenoble Alpes; les gérantes des cinq entreprises de traduction qui ont eu le courage et l'amabilité de partager avec nous leurs savoirs et expériences: Amaïa (Chavanod, Annecy), Altica (Voiron), Atidma (Apprieu), Atit (Grenoble) et Version Internationale (Lyon), énumérées ici dans l'ordre alphabétique; et Iwona Kaliszewska de l'Université de Varsovie pour son autorisation d'utiliser le logiciel EdEt.

\section{NOTES}

1. Le cadre théorique de notre étude, à orientation sémiologique, est esquissé dans Kuznik (2018). Les résultats partiels relatifs à la conceptualisation de l'activité de traduction sont présentés dans Kuznik (soumis[a]), et ceux relatifs à la structure des services de traduction dans Kuznik (sous presse). Ce dernier texte contient aussi plus d'informations sur l'élargissement de l'offre de services de tradition sur le champ de la traduction intralinguale et intersémiotique au moment de la collecte des données (2015) dans les cinq entreprises de traduction françaises. Les détails de l'analyse quantitative portant sur les caractéristiques langagières des entretiens se trouveront dans Kuznik (soumis[b]).

2. Kuznik, Anna (2015a): Theoretical background for studying innovation issues in translationservice activities, non publiée. ( $5^{\text {th }}$ Conference of the International Association for Translation and Intercultural Studies, Belo Horizonte, 7-10 juillet 2015).

3. OCDE/Eurostat (1992/2005): Manuel d'Oslo. Principes directeurs pour le recueil et l'interprétation des données sur l'innovation. $3^{e}$ éd. Paris: OECD Publishing. Consulté le 12 février 2018, <https:// doi.org/10.1787/9789264013124-fr>.

4. Il faut toutefois remarquer que la distinction entre les secteurs primaire, secondaire et tertiaire se voit de plus en plus contestée. Pour les phénomènes de la servicisation de l'industrie et l'industrialisation des services, voir Kuznik (2014: 3-4).

5. Bessant, John et Davies, Andrew (2007): Innovation in Services. DTI occasional paper. Londres: Department of Trade and Industry, Government of United Kingdom. Consulté le 15 février 2019, 
<https://webarchive.nationalarchives.gov.uk/20070701120717/http://www.dti.gov.uk/files/file 39965.pdf $>$.

6. Agrupación de Centros Especializados en Traducción (ACT) (2005): Estudio de mercado de la traducción en España: 2004. Las Rozas/Barcelona: Agrupación de Centros Especializados en Traducción.

7. Même si l'échantillon correspond à un secteur défini du point de vue économique et professionnel (de la même manière que s'il s'agissait de traducteurs professionnels indépendants), cette étude relève de la recherche fondamentale, car elle aborde le sujet vaste mais essentiel de la définition traditionnelle de la traduction et des limites des services de traduction proprement dits, traditionnels, centrés sur la traduction interlinguale.

8. Malgré la rétrogradation de Pluton au rang de planète naine, nous la considérons comme une planète à part entière pour les besoins de la présente étude.

9. Les chiffres entre parenthèses renvoient aux lignes de transcription des entretiens dans le logiciel EdEt.

10. Comité EuRopéen de NORMALISATION (2006): Services de traduction - Exigences requises pour la prestation du service. UNE-EN 15038:2006.

11. Organisation internationale de normalisation (2015): Norme internationale. Service de traduction - Exigences relatives aux services de traduction. ISO 17100:2015 (F).

12. Les liens unissant les milieux professionnels et les universités peuvent être de différents types (la réalisation des projets de recherche communs, la présence de chargés de cours issus du monde professionnel, les stages des étudiants dans des entreprises, l'organisation des séminaires et colloques communs, etc.) mais la portée de cet article ne nous permet pas d'approfondir ce sujet. Les publications de Kuznik (2015b) et Galán-Mañas (2017) en esquissent quelques idées.

\section{RÉFÉRENCES}

Abdallah, Kristiina (2012): Translators in Production Networks. Reflections on Agency, Quality and Ethics. Thèse de doctorat. Joensuu: University of Eastern Finland.

Alonso Lion, Víctor (2014): New Challenges in Interactive Media Localization Projects. In: Carmen Mangiron, Pilar Orero et Minako O’Hagan, dir. Fun for All. Translation and Accessibility Practices in Video Games. Berne: Peter Lang, 269-285.

Bessant, John et TidD, Joe (2007): Innovation and Entrepreneurship. Chichester: John Wiley \& Sons.

Carl, Michael, Bangalore, Srinivas et Schaeffer, Moritz, dir. (2016): New Directions in Empirical Translation Process Research. Exploring the CRITT TPR-DB. Cham: Springer.

Christensen, Tina P. et Schjøldager, Anne (2016): Computer-aided translation tools- the uptake and use by Danish translation service providers. JoSTrans. 25:89-105.

Cravo, Ana et Neves, Josélia (2007) : Action Research in Translation Studies. JoSTrans. 7:97-107.

Dam, Helle V. et Zethsen, Karen K. (2009): Who said low status? A study of factors affecting the perception of translator status. JoSTrans. 12:2-36.

Durand, Jean-Pierre (2004): La Chaîne invisible. Travailler aujourd'hui: flux tendu et servitude volontaire. Paris: Seuil.

EHRENSBERGER-Dow, Maureen (2014): Challenges of translation process research at the workplace. In: Ricardo Muñoz Martí, dir. Minding Translation / Con la traducción en la mente. MonTI. NS(1):355-383.

Ehrensberger-Dow, Maureen et Massey, Gary (2014) : Cognitive ergonomic issues in professional translation. In: John W. Schwieter et Aline Ferreira, dir. The Development of Translation Competence: Theories and Methodologies from Psycholinguistics and Cognitive Science. Newcastle upon Tyne: Cambridge Scholars Publishing, 58-86.

Gambier, Yves (1986): Théorie et pratique: une fausse alternative. Pour un concept dynamique de la traduction. Meta. 31(2):161-172.

Gambier, Yves (2005): Pertinence sociale de la traductologie? Meta. 50(4):8.

GalÁn-Mañas, Anabel (2013): L’apprentissage par projets dans la formation de traducteurs. Une expérience pour professionnaliser l'étudiant. Babel. 59(1):41-56. 
Galán-Mañas, Anabel (2017): Programa para la mejora de la empleabilidad de los egresados en traducción e interpretación. Un estudio de caso. Conexão Letras. 12(17):153-171.

Gieure, Clara et Buendía-Martínez, Inmaculada (2016): Determinants of translation-firm survival: A fuzzy set analysis. Journal of Business Research. 69:5377-5382.

Golden, Sean, Hurtado Albir, Amparo et Piqué, Ramon (1992): La traducció i la interpretació a Catalunya. Noves SL. 17:14-17.

Gouadec, Daniel, dir. (1976): La traduction et l'entreprise. Meta. 21(1).

Gouadec, Daniel (2002): Profession: Traducteur. Paris: La Maison du Dictionnaire.

Gouadec, Daniel (2007): Translation as a Profession. Amsterdam/Philadelphie: John Benjamins.

Hermans, Johan et Lambert, José (1998): From Translation Markets to Language Management: The Implications of Translation Services. Target. 10(1):113-132.

JaKobson, Roman (1959): On linguistic aspects of translation. In: Reuben A. Brower, dir. On Translation. Cambridge: Harvard University Press.

Kou, Xueting (2017): Modelos de calidad de traducción de empresas y de profesionales en la República Popular de China. Thèse de doctorat, non publiée. Bellaterra: Universitat Autònoma de Barcelona.

Kuznik, Anna (2010): El contenido de los puestos de trabajo de los traductores. El caso de los traductores internos en las empresas de traducción de Barcelona. Thèse de doctorat. Bellaterra: Universitat Autònoma de Barcelona.

Kuznik, Anna (2012): El contenido de los puestos de trabajo de los traductores. El caso de los traductores internos en las empresas de traducción de Barcelona. Saarbrücken: Akademikerverlag/Editorial Académica Española.

KuzniK, Anna (2014): Translation as a Paradigmatic Universal, Post-Industrial, KnowledgeBased and Innovative Service. In: Maria Piotrowska et Sergiy Tyupa, dir. Challenges in Translation Pedagogy. inTRAlinea. Numéro special. 13 p. Consulté le 12 février 2018, $<$ http://www.intralinea.org/specials/article/2098>.

KuzNiK, Anna (2015b) : Prácticas, in-service training y transferencia del conocimiento. Formación de traductores e investigación en traducción, en contacto con la realidad del mercado. In: José Jorge Amigo Extremera, dir. Traducimos desde el Sur. Actas del VI Congreso Internacional de la AIETI. (VI Congreso Internacional de la Asociación Ibérica de Estudios de Traducción e Interpretación, Las Palmas de Gran Canaria, 23-25 janvier 2013). Las Palmas de Gran Canaria: Universidad de Las Palmas de Gran Canaria, 566-586.

Kuznik, Anna (2016): La traduction comme travail: perspectives croisées en ergonomie, sociologie et traductologie. ILCEA. 27:16 p. Consulté le 12 février 2018, <http://ilcea.revues. $\operatorname{org} / 4036>$.

Kuznik, Anna (2018): Cadre théorique sémiotique et brève étude exploratoire dans le secteur des services de traduction. In: Florence Lautel-Ribstein et Carmen Pineira-TresmonTANT, dir. Sémantique(s), sémiotique(s) et traduction. Des mots aux actes. 7:493-508.

KuZniK, Anna (sous presse): L’organisation des services dans les PME de traduction françaises. In: Élisabeth Lavault-Olléon et Maria Mizima, dir. Traduction et technologie: regards croisés sur de nouvelles pratiques. Des mots aux actes. 8.

Kuznik, Anna (soumis[a]): Les conceptualisations contemporaines de l'activité de traduction élaborées par les responsables des entreprises de traduction françaises. Między Oryginałem a Przekładem.

Kuznik, Anna (soumis[b]): Comment les responsables de PME de traduction françaises parlentelles de leurs services de traduction? Résultats d'une analyse quantitative exploratoire. Hermes.

KuzniK, Anna et Verd, Joan Miquel (2010): Investigating Real Work Situations in Translation Agencies. Work Content and its Components. Hermes. 44:25-43.

KuzniK, Anna, Verd, Joan Miquel et Olalla-Soler, Cristian (2016): Mixed methods, mixed tools. The use of computer software for integrated qualitative and quantitative analysis. Journal of Research Design and Statistics in Linguistics and Communication Science. 3(1):76109. 
Kwieciński, Leszek, MŁodzińska-Granek, Agnieszka, Rośkiewicz, Wojciech et al. (2015): Przedsiębiorczość akademicka wywodząca się z nauk humanistycznych $i$ społecznych $w$ Niemczech i Polsce. Analiza porównawcza [L'entrepreneuriat académique ressortant des sciences humaines et sociales en Allemagne et en Pologne. Une analyse comparative]. Leipzig/ Vratislavie: Fraunhofer/Uniwersytet Wrocławski.

LAVAult-Olléon, Élisabeth (2011): L'ergonomie, nouveau paradigme pour la traductologie. ILCEA. 14:17 p. Consulté le 12 février 2018, <http://ilcea.revues.org/1078>.

Pedersen, Daniel (2017): Managing Transcreation Projects. Translation Spaces. 6(1):44-61.

Presas Corbella, Marisa, Cid-Leal, Pilar et Torres-Hostench, Olga (2016): Machine translation implementation among language service providers in Spain: a mixed methods study. Journal of Research Design and Statistics in Linguistics and Communication Science. 3(1):126-144.

Risku, Hanna (2014): Translation process research as interaction research: From mental to socio-cognitive processes. In: Ricardo Muñoz Martí, dir. Minding Translation / Con la traducción en la mente. MonTI. NS(1):331-353.

Risku, Hanna, Rogl, Regina et Pein-Weber, Christina (2016) : Mutual Dependencies: Centrality in Translation Networks. JoSTrans. 25:1-22.

Sütiste, Elin et Torop, Peeter (2007): Processual boundaries of translation: Semiotics and translation studies. Semiotica. 163(1):187-207. 